\section{Die Geschichte des Asylanten mit dem Mercedes}

\author{
K. Mohtadi
}

Ich verrate Ihnen kein Geheimnis, haben Sie das vierzigste Lebensjahr erreicht. Sie wissen, vierzig ist eine wichtige Zahl, man erreicht damit die Reife oder zumindest war dies für meine Generation und meinesgleichen so. Man hatte genug Erlebnisse und Erfahrung für Ehe und Beruf. Ein Blick zurückwerfend zieht man Bilanz und schaut sich zielgerichtet orientierend um. Man wird sich auch langsam bewusst, dass es einem da und dort wehtut, der Körper meldet sich. Man ist mit Soma und Psyche am Scheideweg.

Ich hatte schon lange keine Rückenschmerzen mehr. Es waren auch damals keine Schmerzen, die mich plagten, sondern eine Art Brennen, das durch den Schwund der Gelenkknorpel und aneinander reibende abgenutzte Wirbelränder entstand. Der Professor für Orthopädie hatte gemeint, gegen den Gelenkschwund gebe es keine Therapie bis auf Schmerzmittel und Ertragen der Beschwerden. Er verordnete auch keine Physiotherapie. Man bekommt halt, auf das Röntgenbild hinweisend, auch in meinem Alter Rückenbeschwerden, erwiderte er meine Frage. Ich war sehr verblüfft und enttäuscht von meiner Wirbelsäule, der ich jedoch, angesichts des Vertrauens in meine Konstitution, nicht die ganze Schuld anlasten wollte. Ich verdächtigte deswegen die Matratze und das Bett, worauf ich schlief, und zog eine dünne der dicken, weichen aus Schaumgummi und Kamelhaar und die harte Unterlage dem Bett mit Federung und Gelenken vor und verlor gänzlich meine Beschwerden.

Haben Sie schon einmal von einem Bären oder Tiger oder vielleicht einem Affen gehört, Rückenschmerzen gehabt zu haben, ohne jemals auf weicher Matratze und federndem Bett geschlafen zu haben? Der dem Affen am nächsten stehende Vertebrat, der Mensch, mag durch geschichtlich zu kurze Zeit mit aufrechtem Gang Schwierigkeiten haben, mir scheint es jedoch, seine Entfremdung von der Natur bereite ihm mehr Mühe als seine kurze Entwicklungsgeschichte. Der Australopithecus - die älteste bekannte Menschenform - lebte vor drei Millionen bis fünfhunderttausend Jahren. Seine wichtigste Errungenschaft war der aufrechte Gang, wodurch seine Hände frei wurden und seine Hirnentwicklung den Bau von Werkzeugen möglich machte, während der Homo

Korrespondenz:

Dr. med. K. Mohtadi

Kantonale Psychiatrische Klinik

CH-4915 St. Urban sapiens vierzigtausend Jahre alt ist. Weiche Matratze, Federung und flexible Unterlage wären vielleicht für brütende Hühner, aber nicht für den Rücken des Homo sapiens förderlich. Während man also leicht und mit Vorteil auf eine weiche Matratze und auf das federnde Bett verzichten kann, wäre es schwer vorstellbar, sich fortwährend der Schuhe zu entledigen.

Es soll aber nun nicht der Eindruck erweckt werden, ich beabsichtige noch, meinen Hallux valgus den Schuhen zuzuschieben. Diese Gelenkdeformation ist in unserer Familie gut vertreten und ist konstitutionell, genetisch bedingt, die sogenannte Wissenschaft soll behaupten, was sie will. Ich sass also weder wegen meines Rückens noch direkt meines Hallux valgus im Warteraum der Röntgenabteilung im Spital Langenthal. Ein brennender, ziehender Schmerz an der Fusssohle sollte abgeklärt werden. Dort lernte ich sie auch kennen. Sie blätterte eine Illustrierte durch und sah mich neugierig und fragend an, als ich mich hinsetzte; ich guckte sie flüchtig simulierend kühl und neutral an. Wir hatten beide richtig vermutet, es traf zu, sie war eine Landsfrau, mit der ich mich rege zu unterhalten begann. Ich erfuhr, dass sie sich seit knapp einem Jahr mit ihrem Ehemann und der Tochter in der Schweiz aufhält und seit sechs Monaten im Flüchtlingsheim Roggwil wohnt. Sie wollte sich die Lungen durchleuchten lassen wegen eines Fleckens, den sie ohne Beschwerden seit langem auf der Lunge trug und von dessen Existenz sie schon in der Heimat wusste. Sie hatte ihren Mann während des Studiums in den Vereinigten Staaten kennengelernt, beide hatten Elektronik studiert. Mit der Hoffnung auf bessere Zeiten und der Motivation, nun das Gelernte in den Dienst der Heimat zu stellen, waren sie kurz nach ihrem Studiumsabschluss und vor der Revolution in den Iran heimgekehrt. Sie arbeiteten in der Folge in den städtischen Elektrizitätswerken der Stadt Teheran und hatten auch die Wirren der Revolution begeistert miterlebt. Als dann die puritanischen Sitten und das Drängen, nach streng religiösen Regeln zu leben, wuchsen und die Rechtssicherheit nicht mehr gewährleistet war, begann ihr Unbehagen zuzunehmen. Als der Krieg ausbrach und das Leben nach Einfall des Nachbarlandes schwieriger, Grundnahrungsmittel rationiert und Konsumgüter knapp und teuer wurden, steigerte sich ihre Unzufriedenheit.

Die ganz Reichen hatten sich schon während des Aufstandes ins Ausland abgesetzt. Die unzufriedenen, politisch verfolgten oder ungefährdeten Angehörigen der oberen Mittelschicht begaben sich nach dem Umschwung und vor dem Ausbruch des Krieges ins Ausland und schliesslich reisten auch die übrigen Unzufriedenen der Mittelschicht aus und schickten ihre Kinder in die Fremde mit der Hoffnung, sich in irgendeinem europäischen Land oder in den Vereinigten Staaten niederzulassen. Die Unterschicht zahlte für Revolution und Krieg die Zeche; sie verfügte weder über die finanziellen Mittel, noch erfüllte sie sonstige Bedingungen der Emigration.

Die Lage auf den Schlachtfeldern zwang die Verantwortlichen, immer zahlreicher Soldaten und Personal an die Fronten zu schicken. Man begann die 
Reservisten einzuziehen und Personal aus den Staatsangestellten zu rekrutieren. Ihrem Ehemann drohte es, jederzeit einberufen und an die Front geschickt zu werden. Bei der Abneigung gegen die Politik der Regierung und von Natur aus kein Mann des Krieges, entfiel ihm die Bereitschaft, sich der Wahrscheinlichkeit des Einzuges auszusetzen. Er entschloss sich, mit der Familie ins Ausland, in die USA, zu emigrieren. Es galt, das Gesuch um ein amerikanisches Visum im Ausland zu stellen, nachdem die USA seit der Besetzung ihrer Botschaft keine konsiliarische Tätigkeit mehr in Teheran ausübten, und da die Schweiz die USA in Iran vertrat, entschied sich das Ehepaar, mit einem Zwischenhalt in Zürich, das Einreisevisum für die USA zu beantragen. Da aber die Schweizer Botschaft Familien kein Visum für die Schweiz ausstellt, um späteren Asylgesuchen in der Schweiz vorzubeugen, musste sich die Familie anders helfen. Eine "emanzipierte", offensichtlich in der Besorgung von Ausreisepapieren und Organisieren von Fluchtangelegenheiten versierte Frau mit notwendigen Beziehungen besorgte ihnen ein Visum für die Einreise in die Schweiz mit Drum und Dran gegen ein Entgelt von je fünftausend Dollars. In der Schweiz sollten sie dann einen gewissen Diplomaten eines afrikanischen Staates, der einst in Teheran seinem Land diplomatischen Dienst geleistet hatte, aufsuchen, der ihnen bei der Besorgung des amerikanischen Visums behilflich wäre. Sie hatten angenommen, in den USA studiert und mit dortigen Verhältnissen vertraut, quasi Anrecht darauf zu haben, wieder dorthin zurückkehren zu dürfen. Das junge Ehepaar hatte praktisch alle Brücken hinter sich abgebrochen und beinahe für sein Unternehmen Hab und Gut verkauft, um die Reise zu finanzieren. In der Schweiz war dann der sogenannte Diplomat für den Zweck nicht ansprechbar. Das direkte Gesuch der Eheleute um das Visum wurde erwartungsgemäss von der USA-Botschaft abgelehnt. Inzwischen ging die dreimonatige Aufenthaltserlaubnis zu Ende und bald drohte auch ihr übriges Geld auszugehen. Umzug in eine billigere Bleibe, Gesuch um Verlängerung des Aufenthaltes, mit Hoffnung auf ein Wunder, waren die nächsten Schritte. Und schliesslich stellten sie in Bern einen Asylantrag, um nicht mit hängendem Kopf und mittellos die Schweiz Richtung Iran verlassen zu müssen, immer mit dem Hoffnungsschimmer einer Lösung. Sie waren in der Folge über ein Asylantenheim ins Flüchtlingsheim Roggwil gekommen, wo sich noch zwei iranische Familien armenischer Minorität, eine Mutter mit zwei Kindern im Vorschul- und Schulalter und ein 25jähriger Mann aufhielten. Nach verschiedenen Befragungen durch die Behörde bekamen sie wie andere Iraner einen ablehnenden Bescheid, Asyl zu bekommen, mit der Aufforderung, die Schweiz zu verlassen. Inzwischen hatten sie ihre Unterlagen mit Studiums- und Arbeitszeugnissen der australischen Botschaft zugeschickt, um eventuell dorthin auszuwandern. Von dort hatten sie nach zermürbendem Warten und in Verzweiflung über die Notwendigkeit eines Gesundheitszeugnisses Bericht erhalten. Sie sass nun im Warteraum der Radiologie für eine Durchleuchtung.
Sie schüttete das Herz weiter aus. "Und ich schleppe diesen Flecken, Gott weiss, seit wie viel Jahren mit mir herum, und jeder Arzt nimmt diesen Befund ernst und fühlt sich herausgefordert, ohne eine Diagnose stellen zu können. Wie soll ich der australischen Botschaft beweisen, dass dieser Flecken im Röntgenbild ein harmloser Schatten ist, der mich physisch nicht berührt, aber droht, die Zukunft meiner Familie zu bestimmen. Heute bin ich wieder einmal da. Ich lasse mir trotz der Dringlichkeit des Erscheinens eines Funkens in der düsteren Landschaft meines Schicksals nicht mit dem Werkzeug in die Brust schauen. Ich habe Angst und kein Vertrauen in die Ärzte.»

Sie hatte also noch Kraft, nein zu sagen. Ich versprach ihr, dass meine Frau sie anrufen werde, damit sie uns daheim besuchten.

Es braucht wahrlich eine Portion Zivilcourage und eine innere Überwindung und vielleicht auch Mut, gegen die Institution der Ehe kritisch Stellung zu nehmen. Eine Ehe einzugehen scheint mir, sich eine Stahlkette aus Verpflichtungen irdischen und himmlischen Ursprungs anzulegen. Es wird geheiratet der Nachkommenschaft wegen und um der Einsamkeit und dem sexuellen Elend zu entgehen. Und das Elend in der Sexualität betrifft nicht nur den, in dessen Mastdarm der Gerichtsmediziner bei der Obduktion einen dicken Gegenstand findet, der den Darm perforiert hat, und der, um von Mitmenschen nicht verachtet zu werden, aus Schamgefühl den Chirurgen nicht aufgesucht hat - als Homosexueller anonym gelebt, nun auch anonym zu sterben - sich erhängt. Der, dessen Würde in der Triebnot selbst bei Betrachten eines Sexheftchens tangiert wird, befindet sich auch in einem sexuellen Elend.

Die Familie, als Mikrokosmos und Träger der Garantie menschlicher Gesellschaft, soll aus wirtschaftlichen Überlegungen des Mannes, Arbeitskräfte zur Verfügung zu haben, entstanden sein. Es war vielmehr der Sexualtrieb, der den Mann trieb, "das Weib» bei sich zu behalten, und die Frau sah ihre Bedürfnisse erfüllt und begann monatlich zu menstruieren, um durch die permanente Empfänglichkeit ihren Schutz und ihre Existenz zu sichern. Wäre ein phylogenetisch determinierter monatlicher Zyklus geschichtlich nicht jüngeren Datums, würde das physiologische Geschehen keine Beschwerden machen. Die geschichtlich kurze Entwicklungszeit schiene aus dieser Sicht auch der Grund der klimakterischen Störungen zu sein. Wie es auch immer ...

Ich ging also eine Ehe ein und hielt das ständige Meckern und die zur Schau gestellte Überlegenheit mobiler Freunde, Bekannter, Nachbarn nicht mehr aus; ich entschied mich, ein Auto zu kaufen. Der erste Wagen, den mein Vater gekauft hatte, war ein sechsrädriger Lastwagen Marke Chevrolet. Dazu besass er als Transportmittel ein Kontingent von zweiunddreissig Mauleseln, oder waren es nur zwölf, ich weiss es nicht mehr so genau, es sind vierzig Jahre her, und ich sah die Vierbeiner auch nie. Sie brachten aus unwegsamen Wäldern Baumstämme bis zu einer Station im Dschungel, von wo sie eingesägt mit dem 
Lastwagen bis zum Umladeplatz der asphaltierten Strasse transportiert wurden, um mit "Zehnrädern» nach Teheran gefördert, im Lager des Vaters zum Verkauf angeboten zu werden.

Man fuhr nach dem zweiten Weltkrieg auf Teheraner Strassen vorwiegend amerikanische Automarken: Ford, Cadillac, Buick und wie sie sonst alle heissen. Die Bundesrepublik Deutschland galt aber trotzdem als eigentliche Industrienation der Welt, die unsere Sympathie genoss. Der erste Personenwagen den der Vater, Onkel und Schwager gekauft hatte, war ein Mercedes, nicht etwa wegen dem vom CIA gesteuerten Putsch 1952 gegen die Nationalregierung, die Iraner sind weit von solchen Überlegungen entfernt. Man kaufte einfach deutsche Wagen, und mancher damalige Mercedes fährt noch heute mit einer Million Kilometer Fahrt, könnte es der Zähler zeigen, täglich über fünfhundert Kilometer hin und her auf dem Land. Es war also weder ein Prestige- noch ein Statusprodukt, das man kaufte, sondern ein Auto aus einem Land, zu dem man gute Beziehung unterhielt, es wurde auch eine Tradition in der Familie. So kaufte mir mein Cousin in der BRD traditionsgemäss ein vierjähriges 0ccasionsauto Marke Mercedes in einer Mercedes-Garage, in der seine deutsche Frau arbeitete und dessen Verkäufer sein Nachbar war. Alles in allem kam ich nach viel Umständen in den Besitz eines Autos Marke Mercedes.

Wir luden die Asylanten-Landsleute zu uns ein und besuchten sie im Flüchtlingsheim mit unserem "grossen gelben Mercedes». Und keinem steht auf der Stirn, kein Asylant zu sein. Sie wohnten im zweiten Stock, Mitte des Ganges in einem Zweibettzimmer des Flüchtlingsheimes, eines länglichen Blocks hinter einem Ackerland mit gutem Umschwung und einem Balkon mit Aussicht aufs Feld. Ihre vierjährige Tochter sprach bereits rege "Schwyzerdütsch", die Eltern besuchten einen Deutschkurs. Sie wollen nicht in der Schweiz bleiben, müssen aber hier solange verharren, bis sich für sie eine gute Lösung ergibt. Sie leben fortdauernd in Bange und Verzweiflung über die Ungewissheit, was ihnen bevorstünde, es sei zermürbend. "Man hilft uns», erzählten sie. Man behandle die Iraner generell anders, man meine, wir seien auch keine armen Leute. «Wir sind ihnen für ihren Beistand sehr dankbar. Im Schneiderwettbewerb habe ich den ersten Preis, eine Nähmaschine, gewonnen. Mein Mann darf trotz Ablehnung unseres Asylgesuchs arbeiten. Er konnte eine Stelle in einer Elektronikfirma annehmen, wo er in seiner Position eine Stufe tiefer als in der Heimat, aber doch auf seinem Beruf arbeitet.»

Es stellte sich heraus, dass sie eigentlich wenig Ahnung davon haben, keine willkommenen Menschen zu sein und was man über sie in den Zeitungen schreibt und in der Kneipe spricht, und mit welchen Problemen man hierzulande ihretwegen konfrontiert ist.

Die Selbstverständlichkeit des Anrechts auf eine Aufenthaltserlaubnis und Niederlassung in einem westlichen Land schien hintergründig darauf zu beruhen, dass sie aus einem Land stammen, das während siebzig Jahren als vorderster Damm gegen das kommunistische Nachbarland die Interessen des Westens verteidigt hat und heute noch in einer Zeit der Kriegs- und Mangelwirtschaft rund zwei Millionen afghanische Flüchtlinge aus einem kommunistischen Land beherbergt, ohne über einen Bruchteil der Ressourcen westlicher Industrieländer zu verfügen. Ferner setzten manche Länder wie die USA und BRD unter sehr günstigen Bedingungen täglich für über eine Million Dollar bzw. DM Ware in ihrer Heimat ab, was für das letztere Land heute auf über drei Millionen gestiegen ist. Dazu genossen Bürger westlicher Länder in Iran besondere Privilegien und wie heute noch Gastfreundschaft. Im Vordergrund standen aber die sozialpolitischen und rechtlichen Unsicherheiten im Heimatland und internationale Verträge und UNOBestimmungen.

Sie durften auf ihrem Stockwerk in einer gemeinsamen Küche zu bestimmten Zeiten Tee zubereiten. Die Wasserkanne pfiff von weit her. Er lief in die Küche, brachte die braun gefärbte Kanne mit kochendem Wasser. In den Wassergläsern hingen die Teebeutel. Sie entschuldigten sich, uns keinen ordentlichen Tee servieren zu können. Die ölverbrannte Bratpfanne sei oft besetzt und dazu der Belag beschädigt, erzählten sie. Ich schlug ihnen vor, eine gebrauchte, aber gut erhaltene Bratpfanne zu kaufen. Wir würden auch manchmal gebrauchte Artikel im Trödlergeschäft kaufen. In der Schweiz sei es keine Schande, auch für einen Arzt nicht, einen gebrauchten Haushaltartikel zu kaufen. Ich weiss nicht, warum wir nicht auf die Idee gekommen sind, ihnen eine neue Bratpfanne zu schenken. Wahrscheinlich ging es in erster Linie darum, ihnen das Brockenhaus zu zeigen, damit sie auch ohne uns und unser Wissen dort einkaufen konnten.

Nachdem wir sonst mit ansässigen Landsleuten selten Kontakt hatten, pflegten wir eine rege Beziehung zu ihnen und tauchten immer wieder an Wochenenden und Feiertagen unter den Augen der Nachbarn und Angestellten des Heimes dort mit unserem Mercedes auf, nahmen sie mit und brachten sie wieder dorthin zurück.

Erlangt das Auto die Bedeutung eines Prestigeund Liebesobjektes, in das man ausser Geld noch, aus welchen Gründen auch immer, Gefühle übertriebenen Ausmasses investiert, wird man nach einem Totalschaden - ist man physisch davongekommen - psychisch quasi friedhofreif. Setzt man sich in ein Auto, legt man sich eine "Ich-Schale» an, die es zu schützen gibt. Hat man eine oberflächliche Beziehung zum Auto und fährt nicht gern, nimmt man den Zug. So parkierte ich das Auto auf dem Bahnhof, fuhr mit Frau und Tochter nach Bern, wo wir zufällig die Asylantin antrafen. Manche Iranerin könnte man, auch wenn ihre Garderobe von Kleidern zu platzen droht, regelrecht in einem Kaufhaus einsperren, sie würde kaum reklamieren. - Ich verliess die Frauen und schlenderte durch Büchereien und suchte Zeitungen und Zeitschriften mit bescheidenen Auflagen, die man nicht am Kiosk kaufen konnte. Auf der Rückfahrt blieb ich bis Langenthal im Zug stehen, so voll war er von Reisenden, Rucksäcken, Mänteln und 
Koffern. In Langenthal brachte ich mühsam das "Kinderwägeli» durch die Menge und zur Türenge hinaus. Ich war die Zeremonie: Ein- und Aussteigen mit Kinderwagen und Hin- und Herpendeln zur Bahnsteige "St. Urban» gewöhnt. Man half uns immer, im passenden Moment die Geleise zu überqueren; die Unterführung des Bahnhofs Langenthal ist ja nicht kinderwagengängig. Auch dieses Mal gewährte man uns, die Geleise zu überschreiten. Ich nahm die Tochter an die Hand, trug die Säcke, meine Frau schob das Kinderwägeli. Das Auto hatte ich jenseits des Bahnhofs auf dem Parkplatz abgestellt. Ich wartete bis die Frauen kamen. Die Landsfrau mühte sich hinter meiner Frau ab und kam nur langsam voran: In ihren nagelneuen klobigen orthopädischen Schuhen schleuderte sie bei jedem Schritt schwungvoll das rechte Bein nach vorne, streckte den Kopf mit einer halben Drehung nach rechts bei vorgebeugtem Oberkörper. Dabei sprang der Unterschenkel sackmesserartig, und das Bein bildete einen Bogen. Sie stützte sich mit der Hand auf den Oberschenkel, während ihre Handtasche über der Schulter hin- und herschwankte, zog sie sich vorwärts, schleppte das linke Bein nach, verzog flüchtig das Gesicht und demonstrierte darauf lockere Mimik. Sie machte erneut eine Drehung mit dem Oberkörper, hob schwerfällig den rechten Fuss mit dem Klotzschuh, schleuderte ihn seitlich im Halbkreis nach vorne, der Unterschenkel sprang sackmesserartig und streckte sich aus. Sie stützte sich auf dem Oberschenkel mit der Hand bei nach vorne gebeugtem Oberkörper ... die linke Hand trug eine Plastiktasche. Der Bahnhofangestellte linste den Frauen nach, bis sie die Gefahrenzone hinter sich hatten.

Kaum hatte ich die Autotür geöffnet, tauchte eine hagere, flinke Frau im Pensionsalter mit einem grossen Hund auf, die ohne Erklärung aus Distanz die Autonummer zu notieren begann. Ich wurde auf sie durch Hinweis der Landsfrau aufmerksam und wandte mich der älteren Frau zu mit der fragenden Bemerkung: "Sie haben wohl etwas Interessantes entdeckt?» Sie zog sich halb aggressiv, halb abweisend zurück, murmelte etwas französisch von Securitas, dann wieder auf deutsch mit französischem Akzent über "Invalidenparkplätze» und verschwand wie aufgelöst, unheimlich. Ich unterdrückte meine Wut, ich gönnte ihr eigentlich, in ihrem fortgeschrittenen Alter, ihrem Leben - durch Ertappen eines vermeintlichen Parksünders - auf meine Kosten einen Sinn zu geben. Die Landsfrau begann im Auto zu schluchzen: "Ich habe Ihnen noch Unglück gebracht, man hat ihre Autonummer registriert." Ich wusste natürlich nichts von der Existenz der Invalidenparkplätze und weiss noch immer nicht, wo sie sich befinden. Hätte ich auch davon gewusst, ich hätte nicht erahnen können, die behinderte Frau in Bern zu treffen und sie vom Bahnhof heimzufahren. Wie auch immer ... Ich beruhigte sie mit der Mitteilung, dass ich längst registriert sei, hüben und drüben. Sie wussten das nicht? Wenn Sie einmal einem Hilfsbedürftigen unter die Arme gegriffen haben, sind Sie registriert auf Erden und im Himmel.
Ich parkierte den zitronengelben Mercedes vor dem Flüchtlingsheim. Ihr Ehemann kam runter und trug ihre Sachen hinauf. Er war inzwischen ein anderer Mann geworden, wie eine Leiche bewegte er sich fort. Wegen eines Arbeitszeugnisses, das er einer Botschaft zuschicken wollte, schien er mit seinem Arbeitgeber, der mit seiner längeren Anstellung gerechnet hatte, in Zwist geraten zu sein mit der Folge der Kündigung. Müde und spracharm brauchte es ihm die ganzen Kräfte, uns mit freundlicher Gestik zu empfangen.

Der Himmel war bedeckt, eine helle Wolke verzog sich hinter eine schwarze. Es wehte eine leichte Brise und pustete die Federn eines einsamen, verrückt krähenden Raben auf dem Acker vor dem Heim. Aus dem Tonband erklang ein Lied:

Hohe Berge, oh wie

Weite Felder, oh wie

Zwischen den Blüten

duftet's nach frischem Gras, oh wie

Ich suchte in der Gegend

Nach gewissem etwas

Nach einem Lichtloch

Nach einem Obdach vielleicht

Nach einem Traum vielleicht

Nach einem Lächeln vielleicht

Es roch nach Emigration

Ich vernahm nichts

Über die Qualität der Zeit

Und der Erde Vergesslichkeit

Ich schlief auf dem Kissen

Voller Flügelschläge der Schwalben

Hohe Berge, oh wie

Weite Felder, oh wie

Grüne Täler, oh wie

Der Klang der Kuhglocken, oh wie

...

Sie wanderten ein paar Monate später nach Kanada aus. Die Iranerfamilien armenischer Abstammung emigrierten nach den USA. Die bis zu Knochen abgemagerte, lizenzierte Frau, deren Sohn man in der Sekundarschule nicht aufnehmen wollte, reiste mit Kindern nach Frankreich, wo ihr Ehemann inzwischen angekommen war.

Man sprach dann von Asylanten mit dem Mercedes! Ich frage mich, ob dies nicht zu jenen fünfzig Prozent der Missverständnisse des Lebens gehört, mit denen wir zu leben pflegen, und ob überhaupt ein Auto eine Geschichte wert ist?

Am nächsten Tag bekam ich einen Brief von der Mercedes-Garage in Luzern, die alle Details über meinen Wagen wusste, ohne dass ich jemals mit der zu tun gehabt hatte. Der Brief begann mit: "Sehr geehrter Herr ... Sie fahren nicht nur ein Auto, sie fahren einen Mercedes!» Man schickte mir Gratisbillette für den Besuch des Genfer Autosalons. 